Acta Linguistica Petropolitana. 2021. Vol. 17.1. P. 13-35

DOI 10.30842/alp230657371711335

\title{
Рефлексивные местоимения
}

\section{и рефлексивные посессивные аффиксы \\ в быстринском диалекте эвенского языка}

\begin{abstract}
А. О. Бузанов
Национальный исследовательский университет «Высшая школа экономики» (Москва, Россия); anton.buzanov.00@gmail.com
\end{abstract}

Аннотация. В статье упоминаются различные подходы к понятию рефлексивизации, описывается система выражения рефлексивных значений в быстринском диалекте эвенского языка: подробно рассматриваются рефлексивные местоимения, их связь с интенсификаторами, затрагиваются вопросы, связанные с парадигмой рефлексивных посессивных аффиксов. На основе грамматических описаний анализируются данные родственных языков (эвенкийского и нанайского). В статье также рассматриваются несобственно рефлексивные прочтения рефлексивных местоимений и приведен формальный анализ эвенских аргументных рефлексивов в терминах теории связывания.

Ключевые слова: рефлексивы, интенсификаторы, теория связывания, тунгусо-маньчжурские языки.

Благодарности: Исследование осуществлено в рамках Программы фундаментальных исследований НИУ ВШЭ в 2020 году.

\section{Reflexive pronouns and reflexive possessive affixes in the Bystraya dialect of Even}

\section{A. O. Buzanov}

HSE University (Moscow, Russia); anton.buzanov.00@gmail.com

Abstract. This study focuses on different means to license reflexivity in Even and addresses the distribution of reflexive markers within different domains, as well as some semantic properties of these markers. 
The data for this study were collected through fieldwork in villages Anawgaj and Esso of Kamchatka Krai in June-July 2019 and January 2020. Even has different morphosyntactic means to express reflexive meaning that can be divided into two major sets: reflexive pronouns and reflexive possessive suffixes. The connection between reflexive pronouns and intensifiers is also described and a description of the internal structure of reflexive pronouns is proposed.

The paper presents different approaches to reflexivity and the discusses the binding theory. Further, reflexivity-marking strategies in Even are described and their analysis according to their syntactic status is proposed.

Finally, the paper addresses reflexives in Even and the phenomenon of the associative plural they show. Plural pronouns usually denote a group including a focal referent and its contextually specified associates. In case of a number mismatch between the reflexive pronoun and its antecedent in Even, the associates are interpreted as family members, which is well-attested for nouns but has not been described for pronouns. This behavior is unexpected from the typological perspective and deserves further investigation.

Reflexive pronouns in the Bystraya dialect function as reciprocals, while the reciprocal pronouns mentioned in grammatical descriptions have not been attested.

Keywords: reflexives, binding theory, intensifiers, Tungusic languages.

\section{1. Введение}

Эвенский язык, распространенный на территории Сибири и Дальнего Востока России, относится к тунгусо-маньчжурской семье языков. По данным переписи населения 2010 г., на эвенском языке говорят 5700 человек. В рамках работы рассматривается быстринский диалект, распространенный в Быстринском р-не Камчатского края, главным образом, в с. Эссо и Анавгай.

Эвенский язык - это язык с порядком слов SOV и наличием только субъектного согласования. Как и многие другие языки этого региона, эвенский является агглютинативным языком с обилием суффиксов и отсутствием префиксов. Также он является языком номинативно-аккузативного строя. 
Ранее было опубликовано несколько описаний эвенского языка [Богораз 1931; Левин 1936; Цинциус 1947; Новикова 1960; Мальчуков 2008; Pakendorf 2014], однако никто не занимался подробным изучением рефлексивов в этом языке, а также описанием быстринского диалекта. Мое исследование опирается на ряд предшествующих работ; наиболее значимые них — это [Мальчуков 2008] и [Цинциус 1947].

В работе обсуждаются морфологические варианты выражения рефлексивного значения и анализируется их синтаксическое поведение в эвенском языке. Кроме того, описываются несобственно рефлексивные значения рефлексивных маркеров и их морфологическое устройство. Материал для работы был собран в ходе экспедиций в с. Эссо и Анавгай Камчатского края в июне-июле 2019 г. и январе 2020 г.

В Разделе 2 обсуждаются понятие рефлексивизации и основные аспекты теории связывания. В Разделе 3 перечисляются и анализируются способы выражения рефлексивного значения в быстринском диалекте эвенского языка и родственных эвенскому языках, а также кратко затрагивается тема интенсификаторов, очень тесно морфологически связанных с рефлексивными местоимениями. Раздел 4 посвящен синтаксическому поведению анафоров эвенского языка и их нерефлексивным прочтениям. Раздел 5 содержит выводы и обобщения, сделанные в ходе работы.

\section{2. Рефлексивизация и теория связывания}

\section{1. Типология рефлексивов}

Рефлексивные выражения - это выражения, которые обычно используются, чтобы показать, что не-субъект переходного предиката кореферентен субъекту или связан им (в качестве примера можно привести английское выражение $x$-self) [König, Siemund 2000]. В прототипическом случае рефлексивы связываются субъектом, однако это 
не всегда так - они могут быть связаны и другими участниками: косвенным дополнением (см. пример (1), где доступны две интерпретации: себя можно интерпретировать как книги и как Максима - хотя и с приоритетом для связывания субъектом) или топиком, какой бы синтаксической ролью он ни являлся [Koster, Reuland 1991: 181, 203 ]. (1) Книга о открыла Максиму $_{\mathrm{j}}$ себя ${ }_{\mathrm{i} / \mathrm{j}}$ с другой стороны.

В работе [Kemmer 1993] была предложена классификация рефлексивных маркеров по «тяжести». Основным критерием для их различения является формальная сложность устройства маркера. Так, в языках с более чем одним рефлексивным маркером один из них, как правило, морфологически сложнее, чем другие.

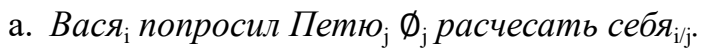

б. Вася попросил Петюо $_{\mathrm{j}} \emptyset_{\mathrm{j}}$ расчесать самого себя ${ }_{\mathrm{j} / *_{\mathrm{i}}}$

Чем сложнее устроен рефлексив, тем правее он находится на шкале от легкого до тяжелого в терминах [Kemmer 1993]. Более тяжелые рефлексивы обычно более локальны (т. е. могут быть связаны в меньшем локальном домене - области, к которой применяются правила связывания) [Haspelmath 2008] (ср. предложения в (2)), поэтому необходимо отделять их от более легких, от которых они отличаются дистрибуцией.

\section{2. Связывание рефлексивов}

В теории управления и связывания [Chomsky 1981] выведены перечисленные ниже условия (принципы) связывания. Они необходимы для того, чтобы описать дистрибуцию трех видов выражений: анафоров, прономиналов и референциальных выражений.

Принцип А. Анафор связан в своем локальном домене.

Принцип В. Прономинал свободен в своем локальном домене.

Принцип С. Референциальное выражение свободно. 
Связывание является важным термином, необходимым для понимания этих принципов.

$\ldots \alpha$ связывает $\beta$, если $\alpha$ и $\beta$ коиндексированы ${ }^{1}$ и $\alpha$ с-командует $\beta$, и нет такой $\gamma$, которая коиндексирована с $\alpha$, с-командует $\beta$ и которой с-командует $\alpha$ [Chomsky 1981: 59].

Несмотря на то, что классическая теория связывания не покрывает всю дистрибуцию прономиналов и анафоров (подробнее см. [Reinhart, Reuland 1993; Reuland 2011; Preminger 2019]), работа выполнена с опорой на эту теорию, т. к. она помогает выявить основные закономерности синтаксического поведения рефлексивов, на основе которых можно проводить более глубокий анализ.

\section{3. Рефлексивы в эвенском языке}

\section{1. Способы выражения рефлексивного значения}

В эвенском отсутствуют глагольные рефлексивные аффиксы, но существует пять различных способов выражения рефлексивного значения:

а. Отсутствие маркирования

b. Рефлексивный посессивный суффикс

c. Рефлексивное местоимение

d. Интенсификатор и рефлексивный посессивный суффикс

е. Интенсификатор и рефлексивное местоимение

В списке стратегии расположены от самой легкой до самой тяжелой, исходя из следующего принципа: ноль легче связанного аффикса, который, в свою очередь, легче полноценного слова.

${ }^{1}$ Коиндексирование - это совпадение референциального индекса, с-командование - отношение структурного приоритета: X с-командует Y тогда и только тогда, когда мать X доминирует над Y, а X не доминирует над Y. 
Известно, что посессивные рефлексивы (типа русского свой) могут подчиняться несколько другим синтаксическим принципам (например, (чаще) нарушать постулаты теории связывания) по сравнению с аргументными (типа русского себя) рефлексивами (см. [Hapspelmath 2008]), поэтому все стратегии рефлексивизации можно разделить на две группы: аргументные (1) и посессивные (2). Таким образом, мы приходим к следующей классификации:

1. а. отсутствие маркирования;

b. рефлексивное местоимение;

c. интенсификатор и рефлексивное местоимение;

2. а. рефлексивный посессивный суффикс;

b. интенсификатор и рефлексивный посессивный суффикс.

Вышеприведенное разделение необходимо, потому что просодически полноценные слова и аффиксы могут подчиняться разным синтаксическим правилам.

Порядок, в котором стратегии будут представлены дальше, не отражает порядок в приведенной классификации, т. к. сведения о некоторых особенностях рефлексивных посессивных суффиксов необходимы для понимания устройства рефлексивных местоимений, а стратегии с интенсификатором (1с и 2b) разделяют некоторые общие свойства.

\section{2. Отсутствие маркирования}

В языках мира глаголы ухода за телом и некоторые глаголы разрушения и созидания склонны к наиболее легкому рефлексивному маркированию [Kemmer 1993]. Часто они совсем не маркированы, как, например, некоторые английские глаголы в (3).

(3) a. Alex washed/undressed/boasts / shook.

б. Alex washed his car/ undressed his son / boasts his knowledge / shook her hand. 
В эвенском также есть несколько глаголов, имеющих как переходное, так и рефлексивное прочтение. В первую очередь это глаголы ухода за телом мыть(ся) (4) и одевать (ся) (5), кроме того, в эту группу входит глагол разрушения резать (ся) (6).

anton awa-d-da- $n^{2}$

A. $\quad$ мыть-PROG-NFUT-3SG

'Антон моет [пол]'. / 'Антон моется'.

(5) etiken tete-d-de-n

старик одевать-PROG-NFUT-3SG

'Старик одевает'. / 'Старик одевается’.

(6) anton mine-ri-n

A. резать-PST-3SG

'Антон порезался'.

Невозможно сказать, присутствует ли в таких глаголах нулевой маркер рефлексивизации или это особый вид лабильности. В рамках этой работы подобные глаголы анализируются как лабильные. Однако в этом случае такой вариант рефлексивизации корректнее называть способом выражения рефлексивного значения, а не отдельной стратегией.

\section{3. Рефлексивные посессивные аффиксы}

Рефлексивные посессивные аффиксы используются, чтобы показать, что посессор не-субъекта глагола кореферентен субъекту или связан им. В эвенском языке таким свойством обладают

2 Для записи данных эвенского языка используется упрощенная графика, принятая в экспедициях НИУ ВШЭ в с. Эссо и Анавгай Камчатского края. Графическая запись не является фонетической транскрипцией в строгом смысле, однако отражает часть фонетических особенностей быстринского говора. Долгота не отмечается, потому что фонетическая разница между краткими и долгими (по словарям) гласными у современных носителей не фиксируется и не осознается информантами, с которыми мы работали. 
суффиксы -i, -i, -j, -mi, -bi, -(w)ur, -bur (см. Таблицу $1^{3}$ и пример (7)), напоминающие по семантике русское свой, норвежское sine, шведское $\sin$ и т. д.

Таблица 1. Рефлексивные посессивные суффиксы в быстринском эвенском языке Table 1. Reflexive possessive suffixes in Bystraya Even

\begin{tabular}{|c|c|c|}
\hline \multirow{2}{*}{ обладатель } & \multicolumn{2}{|c|}{ обладаемое } \\
\cline { 2 - 3 } & $\mathbf{S G}$ & $\mathbf{P L}$ \\
\hline $\mathbf{S G}$ & $-i /-i /-j /-m i$ & $-b i$ \\
\hline PL & \multicolumn{2}{|c|}{$-u r /-w u r /-b u r$} \\
\hline
\end{tabular}
(7) yal-i
mine-ri-wu
рука-POSS.REFL.SG резать-PST-1SG
'Я порезал свою руку'.

B [Haspelmath 2008] и [Despić 2015] утверждается, что язык может иметь рефлексивный посессив, только если он имеет особое рефлексивное местоимение в аргументной позиции. Эвенский язык не нарушает постулируемое правило, т. к. в нем есть рефлексивные местоимения, о которых подробнее будет сказано в Разделе 3.4.

Парадигма эвенских личных посессивных суффиксов представлена в Таблице 2 (с. 21). Личные посессивы различают лицо, число и клюзивность. Характеристики посессора представлены в левой колонке, в правой же приведена фонетическая реализация соответствующего показателя (морфонологические чередования в таблице не отражены).

Рефлексивная посессивность (Таблица 1) устроена совершенно иначе: она не различает лицо, но при этом различает число обладаемого, которое в обычном случае поверхностно выражается суффиксом на самом обладаемом.

Чередование у рефлексивных посессивных суффиксов при множественном посессоре объясняется теми же морфонологическими

\footnotetext{
3 Практически такая же парадигма приводится в [Цинциус 1947: 136].
} 
Таблица 2. Личные посессивные суффиксы в быстринском эвенском Table 2. Personal possessive suffixes in Bystraya Even

\begin{tabular}{|l|l|}
\hline POSS.1SG & $-w u /-b u$ \\
\hline POSs.2SG & $-\check{s}(i)$ \\
\hline POSS.3SG & $-n i$ \\
\hline POSS.1PL.INC & $-t(i)$ \\
\hline POSs.1PL.EXC & - wun/-bun \\
\hline POSS.2PL & $-s ̌ a n$ \\
\hline POSS.3PL & -tan \\
\hline
\end{tabular}

правилами, что и чередование в 1 л. ед. ч. у показателей личной посессивности (после суффикса мн. ч. $-w$ чередуется с $-b$ ). Выбор суффикса в ед. ч. обладаемого и ед. ч. обладателя обусловлен следующими правилами: после гласных аффикс реализуется как $j$, после заднеязычных согласных - как $i$, после основ на - $n-$ как $m i$ (при этом конечное - $n$ основы пропадает), во всех остальных случаях - как $i$. Чередование аффиксов при ед. ч. обладателя $(-i /-\dot{i} /-j /-m i$ vs - $b i)$ не является регулярным, более того - варианты нельзя считать алломорфами, т. к. существуют минимальные пары, ср. (8а) и (8в).
a. polina
or-mi
beri-ri-n
$\Pi$.
олень-POSS.REFL.SG
терять-PST-3SG

'Полина потеряла своего оленя'.

б. polina

ora-l-bi

beri-ri-n

П.

олень-PL-POSS.REFL.PL

терять-PST-3SG

'Полина потеряла своих оленей'

B. polina or-bi

beri-ri-n

П. олень-POSS.REFL.PL[PL $]^{4}$ терять-PST-3SG

'Полина потеряла своих оленей'.

${ }^{4}$ Глосса в квадратных скобках показывает число обладаемого, которое обычно выражается отдельной морфемой (как в (8б)). 
Таким образом, парадигма рефлексивных посессивных аффиксов является асимметричной, что качественно отличает ее от парадигмы личных посессивных аффиксов.

\section{4. Рефлексивные местоимения}

Наиболее прототипической функцией рефлексивных местоимений (как и наиболее распространенной) является маркирование кореференции между субъектом и прямым объектом глагола (см. [König, Siemund 2000; Haspelmath 2008]). Эвенский пример представлен в (9), сама система рефлексивных местоимений представлена в Таблице 3. Прочерки означают, что некоторые формы еще не были собраны.

$$
\begin{array}{lll}
\text { anton } & \text { meni } & \text { eške- } \emptyset-n \\
\text { A. } & \text { SELF.ACC } & \text { хвалить-NFUT-3SG }
\end{array}
$$

'Антон себя хвалит'.

Таблица 3. Склонение рефлексивных местоимений в быстринском эвенском Table 3. Declension of reflexive pronouns in Bystraya Even

\begin{tabular}{|l|c|l|}
\hline & \multicolumn{1}{|c|}{ SG } & \multicolumn{1}{c|}{ PL } \\
\hline ACC & meni & merur \\
\hline INS & - & \multicolumn{1}{c|}{-} \\
\hline DAT & mendi & merdur \\
\hline COM & - & - \\
\hline LOC & mendulej & merdulewur \\
\hline PROL & mendulij & merduliwur \\
\hline DIR & mentekij & mertekiwur \\
\hline DLOC & meneklaj & merklawur \\
\hline ABL & menduki & merdukur \\
\hline ELAT & mengid'i $i$ & - \\
\hline
\end{tabular}


Для сопоставления в Таблице 4 представлена парадигма падежных показателей быстринского эвенского: в первой колонке приведено падежное значение, во второй - реализация после неусеченных основ на - $n$, в третьей — после суффикса мн. ч., а в четвертой в других позициях.

Таблица 4. Падежная система быстринского эвенского

Table 4. Case system of Bystraya Even

\begin{tabular}{|c|c|c|c|c|c|}
\hline & \multicolumn{2}{|c|}{$\begin{array}{c}\text { после } \\
-n\end{array}$} & $\begin{array}{c}\text { после } \\
\text { PL }\end{array}$ & $\begin{array}{c}\text { после } \\
\text { гласного }\end{array}$ & $\begin{array}{c}\text { в других } \\
\text { позициях }\end{array}$ \\
\hline NOM & \multicolumn{5}{|c|}{$-\varnothing$} \\
\hline ACC & \multicolumn{2}{|c|}{$-m$} & $-b u$ & $-u$ & $-w$ \\
\hline INS & \multicolumn{2}{|c|}{$-n^{\prime}$} & $-d^{\prime} i$ & \multicolumn{2}{|c|}{$-\check{c}$} \\
\hline DAT & \multicolumn{5}{|c|}{$-d u$} \\
\hline DES & \multicolumn{2}{|c|}{$-\eta y a$} & \multicolumn{3}{|c|}{$-g a$} \\
\hline $\mathrm{COM}$ & \multicolumn{5}{|c|}{-nun } \\
\hline LOC & \multicolumn{2}{|r|}{-dula } & & \multicolumn{2}{|l|}{$-l a$} \\
\hline PROL & \multicolumn{2}{|r|}{$-d u l i$} & & \multicolumn{2}{|l|}{$-l i$} \\
\hline DIR & \multicolumn{2}{|r|}{$-t a k i$} & & \multicolumn{2}{|l|}{$-t k i$} \\
\hline DLOC & \multicolumn{5}{|c|}{$-k l a$} \\
\hline $\mathrm{ABL}$ & \multicolumn{5}{|c|}{$-d u k$} \\
\hline ELAT & $-\eta i c ̌$ & \multicolumn{4}{|c|}{$-g i \check{c}$} \\
\hline EQU & -nčin & -gačin & \multicolumn{3}{|c|}{-gčin } \\
\hline
\end{tabular}

Существенно, что собранные быстринские данные по склонению рефлексивного местоимения отличаются от данных, представленных в грамматике эвенского языка В. И. Цинциус [Цинциус 1947]. В этом описании форма аккузатива мн. ч. представлена в виде merbur, в собранных же данных - в виде merur (без [b]). Это наблюдение позволило предложить морфологический анализ рефлексивных местоимений. 
Из таблицы видно, что основа рефлексивного местоимения - это men / mer, к которой присоединяется материал, напоминающий суффиксы падежа. Сама основа теп является интенсификатором (подробнее см. в Разделе 3.5). Кроме того, теп является так называемой (терминология [Цинциус 1947]) неусеченной основой на $-n$, одним из свойств которой является выпадение - $n$-, при присоединении суффикса мн. ч. -r.

Все формы, за исключением формы датива, в ед. ч. оканчиваются на - $j$ или - $i$ (последний вариант появляется в результате комбинации конечного - $i$ некоторых падежных суффиксов и $-j$ ), а во мн. ч. на -wur. Сопоставляя данные в Таблицах 1 и 4, можно заметить, что маркеры -j, -i, -wur идентичны рефлексивным посессивным аффиксам, в то время как падежные показатели аналогичны показателям в именной парадигме. Формы датива необычны в том плане, что они оканчиваются на $-d \dot{t}$ и $-d u r$ вместо ожидаемых $-d u-j$ и $-d u-w u r$. Во мн. ч. имеет место обычный для эвенского процесс ослабления аппроксиманта $[\mathrm{w}]$ и его переход в гласный [u]. В ед. ч. же происходит монофтонгизация [uj] в [i]].

Проблемную часть представляют аккузативные формы ${ }^{5}$. В работе [Цинциус 1947] формы аккузатива представлены как meni (SG) и merbur (PL). Форма meni состоит из основы интенсификатора men- и суффикса рефлексивного посессива $-i$, а merbur из основы те(n), суффикса мн. ч. - $r$ и алломорфа рефлексивного посессива после мн. ч. -bur (см. примеры в (10)). Таким образом, оказывается, что аккузатив нарушает выведенное правило образования рефлексивного местоимения, т. к. в этих формах отсутствует морфологически выраженный аффикс аккузатива. Аккузатив, как семантически наименее маркированная форма рефлексива (ввиду отсутствия номинатива), остается немаркированным и на морфологическом уровне (см. [Бойцова 1940: 133] для эвенкийского). Однако в быстринском диалекте форма merbur признается аграмматичной, а вместо нее используется теrur (выбирается алломорф посессивности после согласного) что показано в примере (11).

5 Благодарю анонимного рецензента журнала "Acta Linguistica Petropolitana" за указание на предыдущий неверный анализ. Комментарии рецензента позволили устранить ошибку в конечном варианте статьи. 
(10) a. men-i

INT-POSS.REFL.SG

б. me-r-bur

INT-PL-POSS.REFL.PL

(11)

a. noya-r-tan me-r-ur daš- ̌st-tan

OH-PL-3PL.POSS INT-PL-POSS.REFL.PL укрыть-PST-3PL

б. *noya-r-tan me-r-bur daš-šti-tan

OH-PL-3PL.POSS INT-PL-POSS.REFL.PL укрывать-PST-3PL

'Они укрыли себя'.

Такой выбор алломорфа может быть обусловлен тем, что, несмотря на отсутствие фонетического материала, присутствующий нулевой показатель аккузатива (me-r- $\varnothing-u r$ INT-PL-ACC-POSS.REFL.PL) «блокирует» видимость суффикса мн. ч., оставляя информацию лишь о том, что $-r$ - согласный. Однако предложенный анализ является не более чем предположением, не подкрепленным серьезными наблюдениями. Таким образом, структура рефлексивного местоимения оказывается следующей:

INTENSIFIER + NUMBER OF ANTECEDENT + CASE + REFL. POSSESSIVE SUFFIX

Родственные эвенскому языку эвенкийский и нанайский ведут себя не так. В эвенкийском интенсификаторы и рефлексивы формально идентичны друг другу, как в (12), и похожи по структуре на эвенские рефлексивные местоимения. В нанайском же на синхронном уровне основы рефлексива и интенсификатора различны (13), хотя и восходят к единой основе [Аврорин 1959: 256-258].

Эвенкийский ${ }^{6}$
a. mo-r-tin
hagdi-lō-tin
$h \bar{o}-l$
RFL-PL-PS3PL
большой-LOCALL-PS3PL
очень-PL
'Сами очень большие'.

\footnotetext{
6 Запись производилась в экспедиции под руководством О. А. Казакевич в рамках проекта «Малые языки Сибири: наше культурное наследие». Примеры взяты с сайта http://siberian-lang.srcc.msu.ru/.
} 
б. mo-r-dū-tin d'an-mə hula-wki-l

RFL-PL-DATLOC-PS3PL десяTb-ATR.QUAL оставиTь-PHAB-PL

'Себе десять оставляют' .

Нанайский $^{7}$

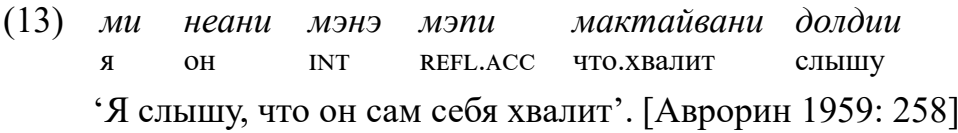

\section{5. Интенсификаторы}

В эвенском языке существуют два интенсификатора теn и men$k e n$ (однако в [Цинциус 1947; Мальчуков 2008] упоминается только $m e n)$, образованных от единой основы men, примеры обоих интенсификаторов представлены в (14).

a. anton konfet-u men karman-dule-j ne-di-n

A. конфета-асс INT карман-LOC-REFL.SG класть-PST-3SG

‘Антон конфету в свой собственный карман положил'.

б. tog men-ken dure-l-li-n

огонь INT-DIM гореть-INCH-PST-3SG

'Огонь сам разгорелся'.

Интенсификаторы способны комбинироваться с аргументными и посессивными рефлексивами. Сочетания интенсификаторов с аргументными рефлексивами ранее не обсуждались в работах, посвященных эвенскому языку. Комбинации подобного рода используются, чтобы обратить внимание слушающего на факт кореферентности субъекта и несубъектного участника. Такие конструкции являются более тяжелыми, т. к. состоят из двух морфологических слов.

${ }^{7}$ Глоссирование проводилось самостоятельно ввиду отсутствия глосс в грамматике В. А. Аврорина. 
Интенсификаторы с рефлексивными посессивными маркерами используются обычно в контрастивных контекстах. Так, (15а) отличается от (15б) тем, что в последнем делается акцент на принадлежности кармана именно Антону, а не кому-то из предыдущего дискурса.

$\begin{array}{llll}\text { a. anton } & \text { konfet-u } & \text { karman-dule-j } & n e-d i-n \\ \text { A. } & \text { конфета-АсC } & \text { карман-LOC-POSS.REFL.SG } & \text { класть-PST-3SG }\end{array}$

'Антон положил конфету в свой карман'.

б. anton konfet-u men karman-dule-j

A. конфета-ACC INT карман-LOC-POSS.REFL.SG

$n e-d i-n$

класть-PST-3SG

'Антон положил конфету в свой собственный карман'.

Модификация рефлексивных местоимений интенсификаторами служит той же цели - демонстрации важности антецедента относительно других участников дискурса, его выделению:
(16)
bi it $\quad i t-w u$
bej-u
men-ken men-di
$1 \mathrm{SG}$ видеть-PST-1SG человек-ACC INT-DIM
INT-DAT.REFL.SG
olla-w
uni-če- $n$
рыба-АCC купить-PPST-Poss.3SG
'Я увидел человека, купившего рыбу для самого себя'.

Кроме семантических различий между конструкцией с интенсификатором и конструкцией без него обнаруживаются и определенные синтаксические. Как показано в [Лютикова 2002; Haspelmath 2008], легкие рефлексивы универсально не более локальны, чем тяжелые. Так, например, разница между русскими себя и сам себя заключается в возможности связывания через границу нефинитной клаузы (ср. (17а) и (17б)).

(17) а. Алексей попросил Антона

б. Алексей иопросил Антона

Подобные различия обнаруживаются и в эвенском, хотя они и не изучены подробно. Некоторые информанты, разрешающие 
связывание через границу целевого конверба, запрещают подобное связывание посредством тяжелого рефлексива:

bi

я

gasči-ri-wu

просить-PST-1SG

(*men) unti-j

(INT)

унты-POSS.REFL.SG

'Я попросил маму взять мои унты'.

Для других нефинитных форм это явление не было изучено и представляет интерес для последующих исследований в этой области.

\section{4. Поведение рефлексивных маркеров}

\section{1. Синтаксический статус эвенских рефлексивных местоимений}

Несмотря на то, что Н. Хомский в [Chomsky 1981] предложил универсальное разделение именных выражений на анафоры, прономиналы и референциальные выражения, существуют языки, в которых один или более из этих типов отсутствует. Так, хантыйский язык не имеет анафоров, которые могут быть связаны локально (этот феномен обсуждается в [Николаева 1995; Volkova, Reuland 2014]). Таким образом, необходимо выяснить, имеются ли в эвенском языке все три типа вышеупомянутых выражений, и удовлетворяют ли рефлексивные местоимения принципам теории связывания.

В эвенском существуют три типа выражений: выражения, которые обязаны быть локально связаны (19а), выражения, которые обязаны быть локально свободны (19б), и выражения, которые должны быть свободны как в локальном домене, так и в нелокальном (19в).

$$
\begin{array}{llll}
\text { a. } \text { etiken }_{\mathrm{i}} \quad{\text { men- } d \dot{t}_{\mathrm{i} / *_{\mathrm{j}}}} \text { olla-w } & \text { ga-di-n } \\
\text { старик себя-DAт } & \text { рыба-АCC } & \text { брать-PST-3SG } \\
\text { 'Старик себе рыбу взял'. } &
\end{array}
$$


$\begin{array}{llll}\text { б. } \text { etiken }_{\mathrm{i}} & \text { noya-du } & \text { olla-w } & \text { ga-di-n } \\ \text { старик } & \text { он-DAт } & \text { рыба-АCC } & \text { брать-PST-3sG }\end{array}$

'Старик ему рыбу взял'.

в. "etiken etiken-di olla-w ga-di-n старик старик-DAT рыба-ACC брать-PST-3SG

'Старик взял рыбу другому старику'.

Ожид.: 'Старик себе рыбу взял'.

Поскольку в предложении (19а) невозможно отличить связывание от кореференции, этот пример не предоставляет достаточно данных, чтобы назвать mendi анафором, управляемым синтаксическими, а не семантическими правилами.

Если рефлексив лицензируется в рефлексивных контекстах и может быть связан кванторными субъектами, такими как никто или каждыц̆, которые не имеют конкретного референта (в реальном мире нет такой сущности, которую можно было бы назвать никто или каждыци), то такие выражения действительно являются анафорами, которые не ориентированы в первую очередь на кореференцию.

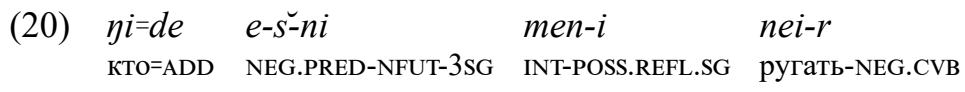 'Никто себя не ругает'.
(21) bejten men-i
ajaw-ra-n
каждый INT-POSS.REFL.SG
любить-NFUT-3SG
'Каждый себя любит'.

Примеры (20) и (21) дают основание говорить о том, что поведение тепi контролируется принципами теории связывания.

\section{2. Нерефлексивные прочтения рефлексивных местоимений}

В быстринском диалекте эвенского языка рефлексивное местоимение используется также для выражения реципрокального значения: 


$\begin{array}{lll}\text { noya-r-tan } & \text { eške-ri-ten } & m e-r \text {-ur } \\ \text { он-PL-POSS.3PL } & \text { хвалить-PST-3PL } & \text { INT-PL-POSS.REFL.PL } \\ \text { 'Они хвалят себя/ друг друга'. }\end{array}$

Как видно, (22) может иметь как рефлексивную интерпретацию, так и реципрокальную. Один из способов анализировать подобное явление - считать, что рефлексив все еще рефлексив, просто его антецедентом является группа референтов: они - это группа, которая хвалит себя, а устройство связей внутри группы может быть любым (см. подробные рассуждения о западнославянских рефлексивах с реципрокальным значением в [Gast, Haas 2008: 339-342]).

Кроме того, эвенские рефлексивы могут использоваться для выражения ассоциативной множественности, что, насколько мне известно, не было ранее зафиксировано ни в одном языке. Такое употребление отмечено в грамматике В. И. Цинциус [Цинциус 1947: 132], однако подробно это явление не обсуждается и там.

Установлено, что для местоимений характерно выражение репрезентативной множественности в терминах [Даниэль 2000]: в этом случае группа, включающая фокусного референта, задается контекстом, т. е. значением местоимения мн. ч. является 'фокусный референт + другие'. Однако в эвенском языке ассоциативная, «семейная» («фокусный референт + его семья») интерпретация является наиболее приемлемой и, как кажется, единственной допустимой в примерах типа (23).

$\begin{array}{llll}\text { etiken } & m e-r-d u-r & \text { olla- } w & g a-d \dot{-}-n \\ \text { старик } & \text { INT-PL-DAT-POSS.REFL.PL } & \text { рыба-АCC } & \text { взять-PST-3SG }\end{array}$

'Старик взял рыбу для себя и своей семьи'.

$$
\begin{array}{llll}
\text { \# } y \text { in } & m e-r-d u-r & \text { olla- } w & g a-d \dot{t}-n \\
\text { собака } & \text { INT-PL-DAT-POSS.REFL.PL } & \text { рыба-АCC } & \text { взять-PST-3SG }
\end{array}
$$

'Собака [в сказке] взяла рыбу для себя и своей семьи'.

Ожид.: 'Собака взяла рыбу для себя и своей стаи / щенков’.

Из примера (24) видно, что в случаях, когда «семейная» интерпретация недоступна, т. е. антецедент не может иметь «семьи», 
ассоциативность практически не лицензируется (в таком случае приходится приравнивать антецедента к существу, которое может иметь семью в традиционном понимании, например - делать сказочным героем). При этом ограничений на антецедент в случаях репрезентативной множественности не появляется, т. к. группа не определяется точно, а задается контекстом.

\section{5. Заключение}

В настоящей работе были описаны пять способов выражения рефлексивного значения в быстринском говоре эвенского языка. Эти способы могут быть разделены на две группы: аргументные (ноль, [интенсификатор +] местоимение) и посессивные рефлексивы ([интенсификатор +] рефлексивный посессивный аффикс). Эксплицитное маркирование отсутствует у некоторых глаголов ухода за телом и глаголов разрушения. Рефлексивные местоимения и рефлексивные посессивные аффиксы могут быть модифицированы интенсификаторами, основа которых также служит для образования рефлексивных местоимений.

Как выяснилось, в быстринском эвенском модификаторами могут выступать два разных интенсификатора (один из которых не упоминается в таком качестве в предыдущих исследованиях), однако дистрибуция двух интенсификаторов пока до конца не изучена. Также эвенский относится к группе языков, в которых интенсификатор формально не совпадает с рефлексивом (в отличие от эвенкийского), но при этом разделяет некоторый морфологический материал (но не в том же смысле, что в нанайском).

Морфологическое устройство рефлексивов практически полностью совпадает с данными из [Цинциус 1947]. Однако парадигма рефлексивных посессивных суффиксов оказалась асимметричной. Аффиксы единичного посессора различаются в зависимости от числа обладаемого. Их чередования нельзя свести к синхронной морфонологии, кроме того, есть контекст, в котором отсутствует аффикс, 
обычно выражающий мн. ч. обладаемого, но при этом использован соответствующий мн. ч. посессивный аффикс (число обладаемого в таком случае интерпретируется как множественное).

Дистрибуция и синтаксический статус рефлексивных местоимений не описывались ранее в грамматиках. В рамках исследования было сделано заключение о том, что местоимения, называемые ранее возвратными, подчиняются классической теории связывания и являются анафорами в терминах [Chomsky 1981]. Также было обнаружено, что более тяжелые рефлексивные конструкции могут быть употреблены, только если локальный домен не превышает размер клаузы (в отличие от более легких, которые допускают связывание через границу целевого конверба).

Несмотря на синтаксическую природу рефлексивных местоимений, некоторые семантические аспекты форм мн. ч. - реципрокальные прочтения и ассоциативная множественность - требуют отдельного описания. Если анализ первых был предложен в работе, то вторая требует детального изучения, которое будет предпринято в дальнейшем.

\section{Список условных сокращений}

1, 2, 3 — 1, 2, 3 лицо; ABL — аблатив; ACC — аккузатив; ADD — аддитив; ATR аттрибутив; COM - комитатив; CVB - конверб; DAT — датив; DES - дезигнатив; DIM - диминутив; DIR — директив; DLOC — директив-локатив; ELAT — элатив; EQU — экватив; ЕXC — эксклюзив; INC — инклюзив; INCH — инхоатив; INS — инструменталис; INT — интенсификатор; LOC — локатив; LOCALL — локатив-аллатив; NEG.PRED — предикативное отрицание; NFUT — небудущее время; NOM — номинатив; PHAB - хабитуальное причастие; PL — множественное число; POSS / PS посессивность; PPST — причастие прошедшего времени; PROG - прогрессив; PROL — пролатив; PST — прошедшее время; PURP — цель; QUAL — качество; REFL / RFL - рефлексив; SELF — основа рефлексива; SG - единственное число.

\section{Литература}

Аврорин 1959 - В. А. Аврорин. Грамматика нанайского языка. Л.: Изд-во Академии наук СССР, 1959. 
Богораз 1931 - В. Г. Богораз. Материалы по ламутскому языку // Тунгусский сборник. 1931. Т. 1. С. 1-106.

Даниэль 2000 - М. А. Даниэль. Типология ассоциативной множественности. Дисс. ... канд. филол. наук. М.: РГГУ, 2000.

Левин 1936 - М. Г. Левин. Эвенки Северного Прибайкалья // Советская этнография. 1936. № 2. С. 71-78.

Лютикова 2002 - Е. А. Лютикова. Когнитивная типология: рефлексивы и интенсификаторы. М.: ИМЛИ РАН, 2002.

Мальчуков 2008 - А. Л. Мальчуков. Синтаксис эвенского языка: структурные, семантические, коммуникативные аспекты. СПб.: Наука, 2008.

Николаева 1995 - И. А. Николаева. Обдорский диалект хантыйского языка. (Mitteilungen der Societas Uralo-Altaica 15; Einheitliche Beschreibung der Dialekte uralischer Sprachen 5). Hamburg: Finnisch-Ugrisches Seminar der Universität Hamburg, 1995.

Новикова 1960 - К. А. Новикова. Очерки диалектов эвенского языка: Ольский говор. М.; Л.: Изд-во Академии наук СССР, Ленинградское отделение, 1960.

Цинциус 1947 - В. И. Цинциус. Очерк грамматики эвенского (ламутского) языка. Л.: Ленинградское отделение Учпедгиза, 1947.

Chomsky 1981 - N. Chomsky. Lectures on Government and Binding. (Studies in Generative Grammar 9). Dordrecht: Foris, 1981.

Despić 2015 - M. Despić. Phases, reflexives, and definiteness // Syntax: A Journal of Theoretical, Experimental and Interdisciplinary Research. 2015. Vol. 18. Iss. 3. P. 201-234. DOI: 10.1111/synt.12031.

Gast, Haas 2008 - V. Gast, F. Haas. On reciprocal and reflexive uses of anaphors in German and other European languages // E. König, V. Gast (eds.). Reciprocals and Reflexives: Theoretical and Typological Explorations. (Trends in Linguistics. Studies and Monographs 192). Berlin; New York: Mouton de Gruyter, 2008. P. $307-346$.

Haspelmath 2008 - M. Haspelmath. A frequentist explanation of some universals of reflexive marking // Linguistic Discovery. 2008. Vol. 6. Iss. 1. P. 40-63. DOI: 10.1349/PS1.1537-0852.A.331.

Kemmer 1993 - S. Kemmer. The Middle Voice. (Typological Studies in Language 23). Amsterdam; Philadelphia: John Benjamins Publishing, 1993. DOI: 10.1075/ts1.23.

Koster, Reuland 1991 - J. Koster, E. Reuland. Long Distance Anaphora. Cambridge: Cambridge University Press, 1991. DOI: 10.1017/CBO9780511627835.

König, Siemund 2000 - E. König, P. Siemund. Intensifiers and reflexives: A typological perspective // Z. Frajzyngier, T. Curl (eds.). Reflexives. Forms and Functions. (Typological Studies in Language 40). Amsterdam; Philadelphia: John Benjamins Publishing, 2000. P. 41-74. DOI: 10.1075/tsl.40.03kon. 
Pakendorf 2014 - B. Pakendorf. System changes in Lamunkhin Ėven: Independent innovations and contact-induced changes // Е. В. Головко (отв. ред.). Системные изменения в языках России. Тезисы докладов международной научной конференции. Санкт-Петербург, 16-18 октября 2014 г. СПб.: НесторИстория, 2014. С. 94-96.

Preminger 2019 - O. Preminger. The Anaphor Agreement Effect: Further evidence against binding-as-agreement. Unpublished manuscript, University of Maryland at College Park, 2019.

Volkova, Reuland 2014 - A. Volkova, E. Reuland. Reflexivity without reflexives? // The Linguistic Review. 2014. Vol. 31. No. 3-4. P. 587-633. DOI: 10.1515/tlr-2014-0012.

\section{References}

Avrorin 1959 - V. A. Avrorin. Grammatika nanayskogo yazyka [Grammar of the Nanai Language]. Leningrad: Publishing House of the USSR Academy of Sciences, 1959.

Bogoraz 1931 - V. G. Bogoraz. Materialy po lamutskomu yazyku [Materials on the Lamut language]. Tungusskiy sbornik. 1931. Vol. 1. P. 1-106.

Chomsky 1981 - N. Chomsky. Lectures on Government and Binding. (Studies in Generative Grammar 9). Dordrecht: Foris, 1981.

Daniel 2000 - M. A. Daniel. Tipologiya assotsiativnoy mnozhestvennosti [Typology of associative plural]. PhD thesis. Moscow: Russian State University for the Humanities, 2000.

Despić 2015 - M. Despić. Phases, reflexives, and definiteness. Syntax: A Journal

of Theoretical, Experimental and Interdisciplinary Research. 2015. Vol. 18. Iss. 3. P. 201-234. DOI: 10.1111/synt.12031.

Gast, Haas 2008 - V. Gast, F. Haas. On reciprocal and reflexive uses of anaphors in German and other European languages. E. König, V. Gast (eds.). Reciprocals and Reflexives: Theoretical and Typological Explorations. (Trends in Linguistics. Studies and Monographs 192). Berlin; New York: Mouton de Gruyter, 2008. P. 307-346.

Haspelmath 2008 - M. Haspelmath. A frequentist explanation of some universals of reflexive marking. Linguistic Discovery. 2008. Vol. 6. Iss. 1. P. 40-63. DOI: 10.1349/PS1.1537-0852.A.331.

Kemmer 1993 - S. Kemmer. The Middle Voice. (Typological Studies in Language 23). Amsterdam; Philadelphia: John Benjamins Publishing, 1993. DOI: 10.1075/tsl.23. Koster, Reuland 1991 - J. Koster, E. Reuland. Long Distance Anaphora. Cambridge: Cambridge University Press, 1991. DOI: 10.1017/CBO9780511627835. 
König, Siemund 2000 —E. König, P. Siemund. Intensifiers and reflexives: A typological perspective. Z. Frajzyngier, T. Curl (eds.). Reflexives. Forms and Functions. (Typological Studies in Language 40). Amsterdam; Philadelphia: John Benjamins Publishing, 2000. P. 41-74. DOI: 10.1075/tsl.40.03kon.

Levin 1936 - M. G. Levin. Evenki Severnogo Pribaykalya [Evenks of the Northern Baikal region]. Sovetskaya etnografiya. 1936. No. 2. P. 71-78.

Lyutikova 2002 - E. A. Lyutikova. Kognitivnaya tipologiya: refleksivy i intensifikatory [Cognitive Typology: Reflexives and Intensifiers]. Moscow: Gorky Institute of World Literature of Russian Academy of Sciences, 2002.

Malchukov 2008 - A. L. Malchukov. Sintaksis evenskogo yazyka: strukturnyye, semanticheskiye, kommunikativnyye aspekty [Syntax of Even: Structural, Semantic, Communicative Aspects]. St. Petersburg: Nauka, 2008.

Nikolayeva 1995 - I. A. Nikolayeva. Obdorskiy dialekt khantyyskogo yazyka [Obdorsk Dialect of the Khanty Language]. (Mitteilungen der Societas Uralo-Altaica 15; Einheitliche Beschreibung der Dialekte uralischer Sprachen 5). Hamburg: Finnisch-Ugrisches Seminar der Universität Hamburg, 1995.

Novikova 1960 - K. A. Novikova. Ocherki dialektov evenskogo yazyka [Essays on the Dialects of the Even Language]. Moscow; Leningrad: Publishing House of the USSR Academy of Sciences, Leningrad branch, 1960.

Pakendorf 2014 - B. Pakendorf. System changes in Lamunkhin Ėven: Independent innovations and contact-induced changes. E. V. Golovko (ed.). Sistemnyye izmeneniya v yazykakh Rossii. Tezisy dokladov mezhdunarodnoy nauchnoy konferentsii. Sankt-Peterburg, 16-18 oktyabrya $2014 \mathrm{~g}$. [System Changes in the Languages of Russia. Abstracts of Reports of the International Scientific Conference. St. Petersburg, October 16-18, 2014]. St. Petersburg: Nestor-Istoriya, 2014. P. 94-96.

Preminger 2019 - O. Preminger. The Anaphor Agreement Effect: Further evidence against binding-as-agreement. Unpublished manuscript, University of Maryland at College Park, 2019.

Tsintsius 1947 - V. I. Tsintsius. Ocherk grammatiki evenskogo (lamutskogo) yazyka [Grammar Sketch of the Even (Lamut) Language]. Leningrad: Leningrad branch of Uchpedgiz, 1947.

Volkova, Reuland 2014 - A. Volkova, E. Reuland. Reflexivity without reflexives? The Linguistic Review. 2014. Vol. 31. No. 3-4. P. 587-633. DOI: 10.1515/tlr2014-0012. 